\title{
On a Class of P-Kenmotsu Manifolds Admitting Weyl-projective Curvature Tensor of Type $(1,3)$
}

\author{
K. L. Sai Prasad ${ }^{1, *}$, S. Sunitha Devi' ${ }^{2}$ G. V.S. R. Deekshitulu ${ }^{3}$ \\ ${ }^{1}$ Department of Mathematics, Gayatri Vidya Parishad College of Engineering for Women, Visakhapatnam, India \\ ${ }^{2}$ Department of Mathematics, Vignan Institute of Information Technology, Visakhapatnam, India \\ ${ }^{3}$ Department of Mathematics, Jawaharlal Nehru Technological University, Kakinada, India \\ *Corresponding author: klsprasad@gvpcew.ac.in
}

Received September 11, 2018; Revised October 23, 2018; Accepted November 24, 2018

\begin{abstract}
We study a class of para-Kenmotsu manifolds admitting Weyl-projective curvature tensor of type $(1,3)$. At the end, it is shown that an n-dimensional $(n>2)$ P-Kenmotsu manifold is Ricci semisymmetric if and only if it is an Einstein manifold.
\end{abstract}

Keywords: para kenmotsu manifold, recurrent manifold, $W_{2}$ - Curvatute tensor, ricci tensor, einstein manifold

Cite This Article: K. L. Sai Prasad, S. Sunitha Devi, and G. V. S. R. Deekshitulu, "On a Class of P-Kenmotsu Manifolds Admitting Weyl-projective Curvature Tensor of Type $(1,3)$." Turkish Journal of Analysis and Number Theory, vol. 6, no. 6 (2018): 155-158. doi: 10.12691/tjant-6-6-2.

\section{Introduction}

In $[1,2]$, Sato introduced the notions of an almost para contact Riemannian manifold. In 1977, Adati and Matsumoto defined para-Sasakian and special para-Sasakian manifolds, which are regarded as a special kind of an almost contact Riemannian manifolds [3]. Para-Sasakian manifolds have been studied by Adati and Miyazawa [4], De and Avijit [5], Matsumoto, Ianus and Mihai [6] and many others. Before Sato, Kenmotsu defined a class of almost contact Riemannian manifolds [7]. In 1995, Sinha and Sai Prasad defined a class of almost para contact metric manifolds namely para-Kenmotsu (briefly P-Kenmotsu) and special para-Kenmotsu (briefly SP-Kenmotsu) manifolds [8].

In 1970, Pokhariyal and Mishra introduced new tensor fields, called $\mathrm{W}_{2}$ and $\mathrm{E}$ tensor fields, on a Riemannian manifold [9]. Later, in [10], Pokhariyal studied some of the properties of these tensor fields on a Sasakian manifold. In 1986, Matsumoto, Ianus and Mihai have extended these concepts to almost para-contact structures and studied para-Sasakian manifolds admitting these tensor fields [6]. These results were further generalised by De and Sarkar, in [5]. Motivated by these studies, in 2015, Sai Prasad and Satyanarayana studied $\mathrm{W}_{2}$-tensor field in an SP-Kenmotsu manifold [11]. In the present work, we investigate a class of para-Kenmotsu manifolds admitting Weyl-projective curvature tensor $\mathrm{W}_{2}$ of type $(1,3)$. The present work is organised as follows: Section 2 is equipped with some prerequisites about P-Kenmotsu manifolds. In Section 3, we define $\mathrm{W}_{2}$-recurrent and semisymmetric para-Kenmotsu manifolds and shown that $\mathrm{W}_{2}$-recurrent para-Kenmotsu manifold is a semisymmetric manifold. Further, it is shown that the curvature of W2-semisymmetric
para-Kenmotsu manifold is constant and hence we establish that a $\mathrm{W}_{2}$-recurrent para-Kenmotsu manifold is an SP-Kenmotsu manifold. Section 4 is devoted to study Ricci semisymmetric P-Kenmotsu manifold.

\section{Preliminaries}

Let $M_{n}$ be an n-dimensional differentiable manifold equipped with structure tensors $(\Phi, \xi, \eta)$ where $\Phi$ is a tensor of type $(1,1), \xi$ is a vector field, $\eta$ is a 1 -form such that

$$
\begin{aligned}
& \eta(\xi)=1 \\
& \Phi^{2}(X)=X-\eta(X) \xi ; \bar{X}=\Phi X .
\end{aligned}
$$

Then the manifold $M_{n}$ is called an almost para-contact manifold.

Let $g$ be a Riemannian metric such that, for all vector fields $X$ and $Y$ on $M_{n}$

$$
\begin{aligned}
& g(X, \xi)=\eta(X) \\
& \Phi \xi=0, \eta(\Phi X)=0, \operatorname{rank} \Phi=n-1 \\
& g(\Phi X, \Phi Y)=g(X, Y)-\eta(X) \eta(Y) .
\end{aligned}
$$

Then the manifold $M_{n}$ [1] is said to admit an almost para-contact Riemannian structure $(\Phi, \xi, \eta, g)$.

In addition, if $(\Phi, \xi, \eta, g)$ satisfies the conditions

$$
\begin{aligned}
& \left(\nabla_{X} \eta\right) Y-\left(\nabla_{Y} \eta\right) X=0 \text {, } \\
& \left(\nabla_{X} \nabla_{Y} \eta\right) Z=[-g(X, Z)+\eta(X) \eta(Z)] \eta(Y) \\
& +[-g(X, Y)+\eta(X) \eta(Y)] \eta(Z),(2.3) \\
& \nabla_{X} \xi=X-\eta(X) \xi \text {, } \\
& \left(\nabla_{X} \Phi\right) Y=-g(X, \Phi Y) \xi-\eta(Y) \Phi X
\end{aligned}
$$


then $M_{n}$ is called para-Kenmotsu manifold or briefly a PKenmotsu manifold [8].

A $P$-Kenmotsu manifold admitting a 1-form $\eta$ satisfying

$$
\begin{aligned}
& \left(\nabla_{X} \eta\right) Y=g(X, Y)-\eta(\mathrm{X}) \eta(\mathrm{Y}) \\
& \left(\nabla_{X} \eta\right) Y=\varphi(\bar{X}, Y)
\end{aligned}
$$

where $\varphi$ is an associate of $\Phi$, is called special para-Kenmotsu manifold or briefly SP- Kenmotsu manifold [8].

Let $\left(M_{n}, g\right)$ be an n-dimensional, $n \geq 3$, differentiable manifold of class $C^{\infty}$ and let $\nabla$ be its Levi-Civita connection. Then the Riemannian Christoffel curvature tensor $\mathrm{R}$ of type $(1,3)$ is given by:

$$
R(X, Y) Z=\nabla_{X} \nabla_{Y} Z-\nabla_{Y} \nabla_{X} Z-\nabla_{[X, Y]} Z
$$

The Ricci operator $S$ and the $(0,2)$ - tensor $S^{2}$ are defined by

$$
g(S X, Y)=S(X, Y)
$$

and

$$
S^{2}(X, Y)=S(S X, Y) .
$$

It is known [8] that in a P-Kenmotsu manifold the following relations hold:

$$
\begin{aligned}
& S(X, \xi)=-(n-1) \eta(\mathrm{X}), \\
& g[R(X, Y) Z, \xi]=\eta[\mathrm{R}(\mathrm{X}, \mathrm{Y}, \mathrm{Z})] \\
& =\mathrm{g}(\mathrm{X}, \mathrm{Z}) \eta(\mathrm{Y})-\mathrm{g}(\mathrm{Y}, \mathrm{Z}) \eta(\mathrm{X}), \\
& R(\xi, X) Y=\eta(\mathrm{Y}) X-g(X, Y) \xi, \\
& R(X, Y, \xi)=\eta(\mathrm{X}) \mathrm{Y}-\eta(\mathrm{Y}) \mathrm{X} ;
\end{aligned}
$$

when $X$ is orthogonal to $\xi$.

An n-dimensional ( $\mathrm{n}>2$ ) Riemannian manifold $M_{n}$ is said to be Einstein manifold if the Ricci curvature tensor $\mathrm{S}(\mathrm{X}, \mathrm{Y})$ of the Levi-Civita connection satisfies the condition

$$
S(X, Y)=\lambda g(X, Y)
$$

where $\lambda$ is a constant.

\section{3. $W_{2}$ - Recurrent P-Kenmotsu Manifolds}

The Weyl-projective curvature tensor $W_{2}$ of type $(1,3)$ of a Riemannian manifold $M_{n}$ with respect to Riemannian connection is given by [9]:

$$
\begin{aligned}
& W_{2}(X, Y, Z, U) \\
& =R(X, Y, Z, U)+\frac{1}{n-1}\left[\begin{array}{l}
g(X, Z) S(Y, U) \\
-g(Y, Z) S(X, U)
\end{array}\right] .
\end{aligned}
$$

Now, we define a $W_{2}$-semisymmetric para-Kenmotsu manifold as:

Definition 3.1: An n-dimensional para-Kenmotsu manifold is called $W_{2}$-semisymmetric if its $W_{2}$-curvature tensor satisfies the condition

$$
R(X, Y) \cdot W_{2}=0
$$

where $R(X, Y)$ is considered to be a derivation of the tensor algebra at each point of the manifold for tangent vectors $X$ and $Y$.

It can be easily shown that on a P-Kenmotsu manifold the $W_{2}$-curvature tensor satisfies the condition

$$
W_{2}(X, Y, Z, \xi)=0 \text {. }
$$

Further, we define a $W_{2}$-recurrent para-Kenmotsu manifold as:

Definition 3.2: An n-dimensional para-Kenmotsu manifold with respect to the Levi-Civita connection is called $W_{2}$-recurrent manifold if its $W_{2}$-curvature tensor satisfies the condition

$$
\left(\nabla_{U} W_{2}\right)(X, Y) Z=A(U) W_{2}(X, Y) Z,
$$

where $A$ is some non-zero 1 -form.

Now, let us establish a relation between $W_{2}$-recurrent and $W_{2}$-semisymmetric para-Kenmotsu manifolds.

For that, let us suppose that $W_{2} \neq 0$. Now, we define a function by

$$
f^{2}=g\left(W_{2}, W_{2}\right) .
$$

Using the fact that $\nabla_{U} g=0$, from (3.5) we get $2 f(U f)=2 f^{2}(A(U))$.

Since $f \neq 0$, we have

$$
U f=f(A(U)) .
$$

Then, from (3.6), we get

$$
X(U f)=\frac{1}{f}(X f)(U f)+(X A(U)) f
$$

and hence, we have

$$
X(U f)-U(X f)=[X A(U)-U A(X)] f .
$$

Therefore,

$$
\begin{aligned}
& \left(\nabla_{X} \nabla_{U}-\nabla_{U} \nabla_{X}-\nabla_{[X, U]}\right) f \\
& =[X A(U)-U A(X)-A([X, U])] f \\
& =2[d A(X, U)] f .
\end{aligned}
$$

Since the left hand side of (3.9) is zero and $f \neq 0$, we deduce that $d A(X, Y)=0$ and it shows that the 1 -form $\mathrm{A}$ is closed.

Then from (3.4), we get that

$$
\begin{aligned}
& \left(\nabla_{V} \nabla_{U} W_{2}\right)(X, Y) Z \\
& =[V A(U)+A(V) A(U)] W_{2}(X, Y) Z,
\end{aligned}
$$

and hence, we get that

$$
\begin{aligned}
& \left(\nabla_{V} \nabla_{U} W_{2}\right)(X, Y) Z-\left(\nabla_{U} \nabla_{V} W_{2}\right)(X, Y) Z \\
& -\left(\nabla_{[U, V]} W_{2}\right)(X, Y) Z \\
& =2 d A(V, U) W_{2}(X, Y) Z=0
\end{aligned}
$$


i.e., $R(V, U) \cdot W_{2}=0$, where $R(V, U)$ is considered to be a derivation of tensor algebra at each point of the manifold for the tangent vectors $\mathrm{V}$ and $\mathrm{U}$.

This shows that a $W_{2}$-recurrent P-Kenmotsu manifold is $W_{2}$-semisymmetric and hence we state that:

Theorem 3.1: A $W_{2}$-recurrent para-Kenmotsu manifold is $W_{2}$-semisymmetric.

Further we determine the curvature value of $W_{2}$-semisymmetric P-Kenmotsu manifold.

From (3.2), we have

$$
\begin{aligned}
& R(X, Y) W_{2}(Z, U) V-W_{2}(R(X, Y) Z, U) V \\
& -W_{2}(Z, R(X, Y) U) V-W_{2}(Z, U) R(X, Y) V=0,
\end{aligned}
$$

which implies

$$
\begin{aligned}
& g\left(R(X, Y) W_{2}(Z, U) V, \xi\right) \\
& -g\left(W_{2}(R(X, Y) Z, U) V, \xi\right) \\
& -g\left(W_{2}(Z, R(X, Y) U) V, \xi\right) \\
& -g\left(W_{2}(Z, U) R(X, Y) V, \xi\right)=0 .
\end{aligned}
$$

By putting $X=\xi$ in the above equation, we get

$$
\begin{aligned}
& R\left((\xi, Y) W_{2}(Z, U) V, \xi\right) \\
& -W_{2}(R(\xi, Y) Z, U, V, \xi) \\
& -W_{2}(Z, R(\xi, Y) U, V, \xi) \\
& -W_{2}(Z, U, R(\xi, Y) V, \xi=0) .
\end{aligned}
$$

Now, by using (2.8) and (3.3), the above equation reduces to:

$$
\eta(Y) \eta\left(W_{2}(Z, U) V\right)-g\left(Y, W_{2}(Z, U) V\right)=0 .
$$

Again on using (3.3), we get that $W_{2}(Z, U, V, Y)=0$. Therefore, from (3.1) we have

$$
R(X, Y, Z, V)=\frac{1}{n-1}\left[\begin{array}{l}
g(Y, Z) S(X, V) \\
-g(X, Z) S(Y, V)
\end{array}\right] .
$$

On contracting the above equation, we get

$$
S(Y, Z)=\frac{r}{n} g(Y, Z) .
$$

Then, from equations (3.16) and (3.17), we have

$$
R(X, Y, Z, V)=\frac{r}{n(n-1)}\left[\begin{array}{l}
g(Y, Z) g(X, V) \\
-g(X, Z) g(Y, V)
\end{array}\right] .
$$

This shows that the curvature of $W_{2}$-semisymmetric P-Kenmotsu manifold is constant.

As it is known [8] that a P-Kenmotsu manifold with constant curvature is an SP-Kenmotsu manifold and using the above shown result, we state that:

Theorem 3.2: $\mathrm{A} \quad \mathrm{W}_{2}$-semisymmetric $\mathrm{P}$-Kenmotsu manifold is an SP-Kenmotsu manifold.

Therefore, form theorems (3.1) and (3.2), we have the following result:

Theorem 3.3: $A W_{2}$-recurrent P-Kenmotsu manifold is an SP-Kenmotsu manifold.

\section{Ricci Semisymmetric Para-Kenmotsu Manifolds}

Definition 4.1: An n-dimensional Riemannian manifold is said to be Ricci semisymmetric if its Ricci tensor $S(X, Y)$ of the Levi-Civita connection satisfies the condition

$$
R(X, Y) . S=0 .
$$

Theorem 4.1: An n-dimensional $(\mathrm{n}>2)$ P-Kenmotsu manifold $M_{n}$ is Ricci semisymmetric if and only if it is an Einstein Manifold.

Proof: Let us suppose that a P-Kenmotsu manifold be Ricci semisymmetric. Then from (4.1), we have

$$
S(R(X, Y) U, V)+S(U, R(X, Y) V)=0 .
$$

By putting $X=\xi$ in (4.2), we get

$$
S(R(\xi, Y) U, V)+S(U, R(\xi, Y) V)=0 .
$$

Now by using the equations (2.8) (a) and (2.8) (c), the above equation reduces to

$$
\begin{aligned}
& \eta(U) S(Y, V)+(n-1) \eta(V) g(Y, U) \\
& +\eta(V) S(U, Y)+(n-1) \eta(U) g(Y, V)=0 .
\end{aligned}
$$

Again by putting $X=\xi$ in (4.4), we get

$$
S(Y, V)=-(n-1) g(Y, V) \text {. }
$$

This proves that the manifold $M_{n}$ is an Einstein manifold.

As an every Einstein manifold is Ricci semisymmetric, the converse of the theorem is trivial.

This completes the proof.

\section{Statement of Competing Interests}

The authors declare that there is no conflict of interests regarding the publication of this paper.

\section{Acknowledgements}

The authors acknowledge Prof. Kalpana of Banaras Hindu University, Varanasi for her valuable suggestions in preparation of the manuscript.

\section{References}

[1] Sato, I, On a structure similar to the almost contact structure, Tensor (N.S.), 30, 219-224, 1976.

[2] Sato, I, On a structure similar to the almost contact structure II, Tensor (N.S.), 31, 199-205, 1977.

[3] Adati, T. and Matsumoto, K, On conformally recurrent and conformally symmetric P-Sasakian Manifolds, TRU Math., 13, 2532, 1977.

[4] Adati, T. and Miyazawa, T, On P-Sasakian manifolds admitting some parallel and recurrent tensors, Tensor (N.S.), 33, 287-292, 1979.

[5] De, U. C. and Avijit Sarkar, On a type of P-Sasakian manifolds, Math. Reports, 11(2), 139-144, 2009. 
[6] Matsumoto, K., Ianus, S. and Ion Mihai, On P-Saskian manifolds which admit certain tensor fields, Publ. Math. Debrecen, 33, 6165, 1986.

[7] Kenmotsu, K, A class of almost contact Riemannian manifolds, Tohoku Math. Journal, 24, 93-103, 1972.

[8] Sinha, B. B. and Sai Prasad, K. L, A class of almost para contact metric Manifold, Bulletin of the Calcutta Math. Soc., 87, 307-312, 1995.
[9] Pokhariyal, G. P. and Mishra, R. S, The curvature tensors and their relativistic significance, Yokohoma Math. J., 18, 105-108, 1970.

[10] Pokharial, G. P, Study of a new curvature tensor in a Sasakian manifold, Tensor (N.S.), 36, 222-225, 1982.

[11] Sai Prasad, K. L. and Satyanarayana, T, Some curvature properties on a Special paracontact Kenmotsu manifold with respect to Semisymmetric connection, Turkish Journal of Analysis and Number Theory, 3(4), 94-96, 2015. 